\section{(6) OPEN ACCESS}

\title{
Orthostatic blood pressure recovery patterns in suspected syncope in the emergency department
}

\author{
Veera K van Wijnen, ${ }^{1}$ Dik Ten Hove, ${ }^{1}$ Reinold O B Gans, ${ }^{1}$ Wybe Nieuwland, ${ }^{2}$ \\ Arie M van Roon, ${ }^{1}$ Jan C Ter Maaten, ${ }^{1}$ Mark P M Harms ${ }^{1}$
}

\begin{abstract}
- Additional material is published online only. To view please visit the journal online (http://dx.doi.org/10.1136/ emermed-2017-207207).

1 Department of Internal, Emergency and Vascular Medicine, University of Groningen, University Medical Center Groningen, Groningen, The Netherlands ${ }^{2}$ Department of Cardiology, University of Groningen, University Medical Center Groningen, Groningen, The Netherlands
\end{abstract}

\section{Correspondence to} Dr Mark P M Harms, Department of Internal, Emergency and Vascular Medicine, University Medical Center Groningen, University of Groningen, Groningen 9713 GZ, The Netherlands;

m.p.m.harms@umcg.nl

Received 25 September 2017 Accepted 22 December 2017 Published Online First 24 January 2018
Check for updates

To cite: van Wijnen VK, Ten Hove D, Gans ROB, et al. Emerg Med J 2018;35:226-230

\begin{abstract}
Introduction Orthostasis is a frequent trigger for (pre)syncope but some forms of orthostatic (pre) syncope have a worse prognosis than others. Routine assessment of orthostatic BP in the ED can detect classic orthostatic hypotension, but often misses these other forms of orthostatic (pre)syncope. This study aimed to determine the frequency of abnormal orthostatic BP recovery patterns in patients with (pre)syncope by using continuous non-invasive BP monitoring.
\end{abstract}

Methods We performed a prospective cohort study in suspected patients with (pre)syncope in the ED of a tertiary care teaching hospital between January and August 2014. Orthostatic BP was measured during the active lying-to-standing test with Nexfin, a continuous non-invasive finger arterial pressure measurement device. Orthostatic BP recovery patterns were defined as normal $\mathrm{BP}$ recovery, initial orthostatic hypotension, delayed BP recovery, classic orthostatic hypotension and reflexmediated hypotension.

Results of 116 patients recruited, measurements in 111 patients (age 63 years, 51\% male) were suitable for analysis. Classic orthostatic hypotension was the most prevalent abnormal BP pattern (19\%), but only half of the patients received a final diagnosis of orthostatic hypotension. Initial orthostatic hypotension and delayed BP recovery were present in $20 \%$ of the patients with (pre)syncope of whom $45 \%$ were diagnosed as unexplained syncope. Reflex-mediated hypotension was present in $4 \%$ of the patients.

Conclusion Continuous non-invasive BP measurement can potentially identify more specific and concerning causes of orthostatic (pre)syncope. Correct classification is important because of different short-term and longterm clinical implications.

\section{INTRODUCTION}

Suspected (pre)syncope in the ED is a difficult clinical problem and many patients are discharged without a diagnosis (17\%-33\%). ${ }^{1}$ Orthostasis is a frequent trigger for (pre)syncope, but it is underappreciated that there are several forms of orthostatic (pre)syncope, associated with different underlying problems and degree of risk. ${ }^{2}$ Initial orthostatic hypotension, delayed BP recovery, classic orthostatic hypotension and reflex-mediated hypotension are all recognised causes for (pre)syncope and falls, but delayed BP recovery and classic orthostatic hypotension present a higher risk because of the association with cardiovascular morbidity and mortality. $^{3}$

\section{Key messages}

What is already known on this subject

- Classic orthostatic hypotension may be diagnosed with traditional orthostatic BP measurement. However, initial orthostatic hypotension, delayed BP recovery and reflexmediated hypotension, forms of orthostasis that are more frequently associated with falls and mortality, are often missed using standard orthostatic BP testing.

What this study adds

- This prospective cohort study used a continuous, non-invasive BP monitoring to determine the frequency of abnormal orthostatic BP recovery patterns. While classic orthostatic hypotension was most prevalent in suspected patients with (pre)syncope, initial orthostatic hypotension and delayed $\mathrm{BP}$ recovery were present in a considerable number of patients. Continuous non-invasive BP measurement can potentially identify more specific and concerning causes of (pre)syncope.

Classic orthostatic hypotension is a common (4\%-24\%) cause of (pre)syncope and can be detected during routine orthostatic BP measurement. ${ }^{1}$ However, the inability of the oscillometric orthostatic BP measurement to measure rapid BP changes limits further evaluation of other forms of orthostatic (pre)syncope. Moreover, the high rate of unexplained syncope justifies new evaluation strategies. $^{1}$

Continuous non-invasive BP measurement can differentiate between abnormal orthostatic BP recovery patterns. Continuous non-invasive finger arterial pressure measurement is based on dynamic (pulsatile) unloading of the finger arterial walls using an inflatable finger cuff with built-in photoelectric plethysmograph. ${ }^{4}$ From the finger waveform, heart beats are detected and systolic, diastolic and mean pressure and pulse rate are output in a beat-to-beat mode. The device has extensively been validated as a reliable method to track orthostatic changes in $\mathrm{BP}^{4}$

As orthostasis is a frequent trigger for (pre) syncope, we aimed to determine the frequency of different orthostatic BP recovery patterns in patients with (pre)syncope in the ED that cannot be captured with intermittent measurements such as those with standard oscillometric BP cuffs. 


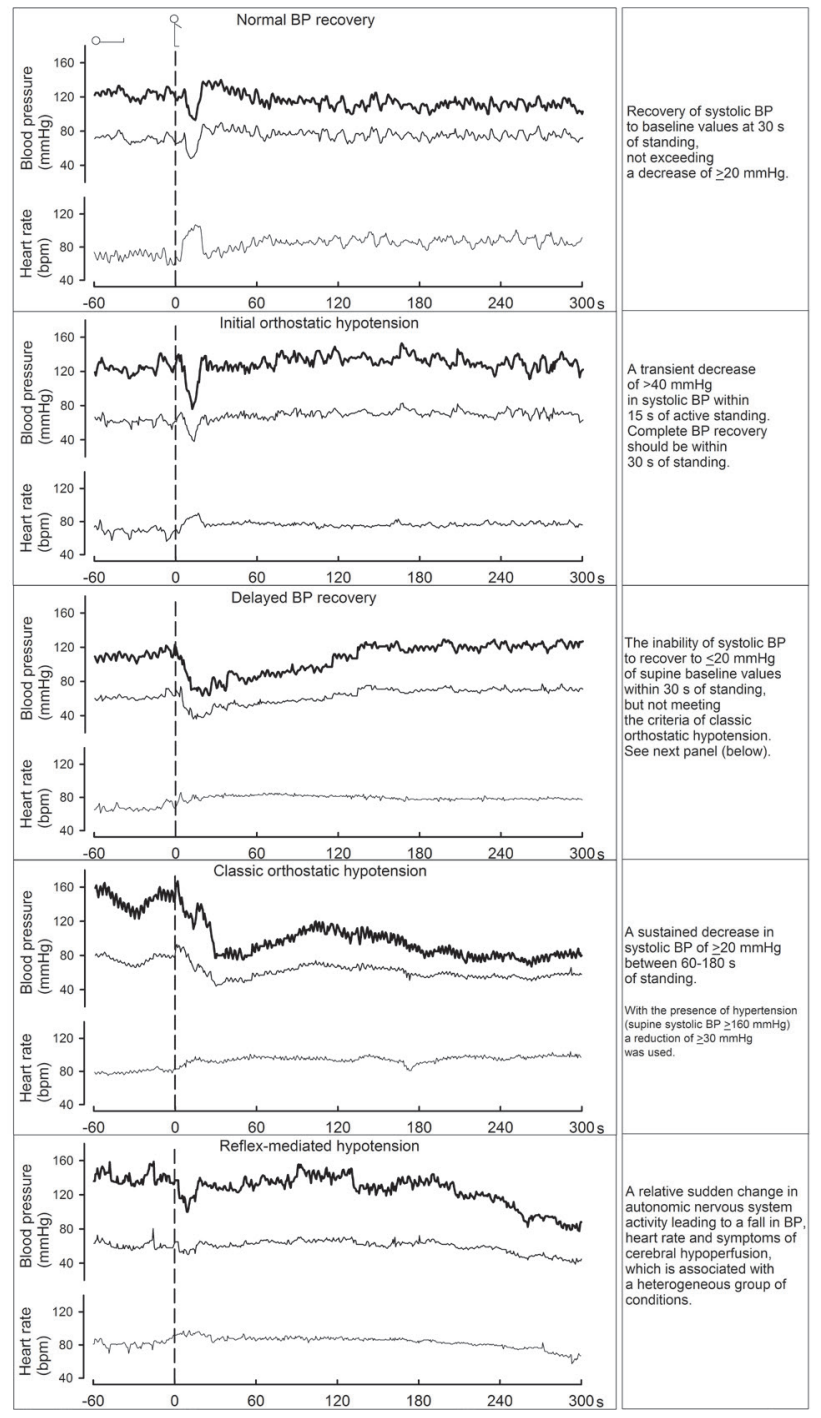

Figure 1 Definitions and illustrations of five distinct orthostatic $\mathrm{BP}$ recovery patterns on standing. Continuous non-invasive BP measurement during active lying-to-standing test in five suspected patients with (pre)syncope. Illustrated are the last $60 \mathrm{~s}$ in supine position and $300 \mathrm{~s}$ of standing. Systolic and diastolic BP and HR are shown.

\section{METHODS}

\section{Setting}

This prospective cohort study was conducted in the ED of a tertiary care teaching hospital, the University Medical Center Groningen, between January and August 2014.

\section{Participants}

All consecutive suspected patients with (pre)syncope older than 18 years visiting the ED Monday to Friday during regular working hours (08:00-18:00) were eligible. Patients were referred by ambulance emergency services, by general practitioner, by specialists or self-referred within 1 hour after the (pre) syncope episode. Patients were excluded if they were not able to stand for $5 \mathrm{~min}$, were haemodynamically unstable (systolic BP $<90 \mathrm{~mm} \mathrm{Hg}$ ), in need of immediate treatment or if informed consent was impaired by a cognitive disorder.

Syncope was defined as a transient loss of consciousness due to transient global cerebral hypoperfusion characterised by rapid onset, short duration and spontaneous complete recovery. ${ }^{1}$ Presyncope was defined as the feeling of almost losing consciousness with similar prodromal symptoms as in syncope. Patients with presyncope are as likely as patients with syncope to experience critical interventions or adverse events like bradydysrhythmia and haemorrhage. ${ }^{56}$

\section{Procedures and variables}

After initial evaluation by the attending physician, patients were approached to participate in the study. Verbal informed consent was obtained from all patients. The orthostatic BP measurement was performed with Nexfin (BMEYE, Edwards Lifesciences, Irvine, California, USA), a continuous non-invasive finger arterial pressure measurement device. The finger cuff, a height correction unit which corrects for movements of the finger when the hand is not kept at heart level, and the wrist-worn unit are connected to a primary unit which holds the air pump, electronics and computer. At the start of the orthostatic BP measurement, patients were lying in supine position; the Nexfin wrist-worn unit was placed around the wrist and the appropriately sized finger cuff was placed around the middle finger. BP was measured continuously during $5 \mathrm{~min}$ of supine rest and 5 min after active standing up. Subsequently patients were asked whether they experienced any symptoms during standing, such as light-headedness, dizziness or blurred vision. Further details on the device and active stand protocol are available in the online Supplementary file 1. The orthostatic BP recovery patterns were defined according to a recent review (figure 1). ${ }^{2}$

Attending physicians in the ED work according to the syncope guideline of the European Society of Cardiology. ${ }^{1}$ No specific criteria with regard to the diagnosis were given to the attending physicians. The attending physician was unaware of conclusive information obtained with continuous non-invasive BP measurement because this was not available at the time of the visit. The attending physician's final diagnosis was obtained from the discharge letter. Patient's demographic features, comorbidities and medication were also obtained from the discharge letter. These features were chosen because of the known association with abnormal orthostatic BP recovery patterns. ${ }^{7}$

The primary outcome of the study was the frequency of the different abnormal orthostatic BP recovery patterns. Secondary outcome was a comparison of the orthostatic BP patterns with the final diagnosis of the (pre)syncopal episode in the ED discharge letter.

\section{RESULTS}

One hundred and sixteen patients consented to participate. Measurements were suitable for analysis in 111 patients (median age $63 \pm 30$ years, $51 \%$ male). Five measurements were excluded due to poor quality signal (artefacts) or signal interruptions. Sixty-six (59\%) patients were referred because of syncope and $45(41 \%)$ patients because of presyncope. Fifty-seven per cent of the 111 patients had a normal BP recovery, 7\% had initial orthostatic hypotension, 13\% delayed BP recovery, 19\% classic orthostatic hypotension and 4\% had reflex-mediated hypotension (table 1). Haemodynamic profiles of the different patterns are presented in figure 2.

Seven out of $45(16 \%)$ patients classified by the attending physician as vasovagal syncope had classic orthostatic hypotension with continuous BP measurement (table 2). In patients classified as orthostatic hypotension by the attending physician, $11 / 18(61 \%)$ had a positive test for classic orthostatic hypotension. In patients with a delayed BP recovery, 6/14 (43\%) were 
Table 1 Patient's characteristics based on the orthostatic BP recovery patterns

\begin{tabular}{|c|c|c|c|c|c|c|}
\hline & All patients $(n=111)$ & $\begin{array}{l}\text { Normal BP } \\
\text { recovery }(n=63)\end{array}$ & $\begin{array}{l}\text { Initial orthostatic } \\
\text { hypotension }(n=8)\end{array}$ & $\begin{array}{l}\text { Delayed } B P \\
\text { recovery }(n=14)\end{array}$ & $\begin{array}{l}\text { Classic orthostatic } \\
\text { hypotension }(n=21)\end{array}$ & $\begin{array}{l}\text { Reflex-mediated } \\
\text { hypotension }(n=5)\end{array}$ \\
\hline \multicolumn{7}{|l|}{ Demographics } \\
\hline Male, n (\%) & $56(51)$ & $30(48)$ & $3(38)$ & $8(57)$ & $11(52)$ & $4(80)$ \\
\hline Age, in years & $63(30)$ & $59(37)$ & $62(42)$ & $73(21)$ & $62(27)$ & $66(45)$ \\
\hline \multicolumn{7}{|l|}{ Medical history, n (\%) } \\
\hline Hypertension & $36(32)$ & $18(29)$ & $1(12)$ & $10(71)$ & $6(29)$ & $1(20)$ \\
\hline Myocardial infarction & $14(13)$ & $8(13)$ & - & $4(29)$ & 1 (5) & $1(20)$ \\
\hline Atrial fibrillation & $20(18)$ & $7(11)$ & $3(38)$ & $5(36)$ & $5(24)$ & - \\
\hline Diabetes mellitus & $15(14)$ & $9(14)$ & $1(12)$ & $2(14)$ & $3(14)$ & - \\
\hline \multicolumn{7}{|l|}{ Medication, n (\%) } \\
\hline Beta blocker & $31(28)$ & $20(32)$ & $2(25)$ & $4(29)$ & $5(24)$ & - \\
\hline ACE-inhibitor & $20(18)$ & $12(19)$ & - & $3(21)$ & $4(19)$ & $1(20)$ \\
\hline Angiotension II antagonist & $14(13)$ & $4(6)$ & $2(25)$ & $5(36)$ & $2(10)$ & $1(20)$ \\
\hline Calcium antagonist & $18(16)$ & $6(10)$ & $2(25)$ & $6(43)$ & $3(14)$ & $1(20)$ \\
\hline Diuretics & $27(24)$ & $18(29)$ & - & $5(36)$ & $4(19)$ & - \\
\hline \multicolumn{7}{|c|}{ Symptoms of orthostatic intolerance, $\mathrm{n}(\%)$} \\
\hline During orthostatic test & $42(38)$ & $18(29)$ & $3(38)$ & $5(36)$ & $11(52)$ & $5(100)$ \\
\hline History of (pre)syncope & $77(69)$ & $44(70)$ & $5(62)$ & $9(64)$ & $16(76)$ & $3(60)$ \\
\hline Admission, $\mathrm{n}(\%)$ & $21(19)$ & $8(13)$ & $1(12)$ & $6(43)$ & $4(19)$ & $2(40)$ \\
\hline
\end{tabular}

The dichotomous values are given in number of patients $(\mathrm{n})$ with \%. Age: median with IQR.

classified as unexplained syncope. The presumed aetiology of the cardiac causes were cardiac ischaemia $(n=1)$ and arrhythmia $(n=9)$. Arrhythmia was further subdivided into non-sustained ventricular tachycardia $(n=1)$, ventricular fibrillation followed by implantable cardioverter defibrillator-discharge $(n=1)$, bradycardia with atrioventricular block $(\mathrm{n}=3)$ and atrial flutter/atrial
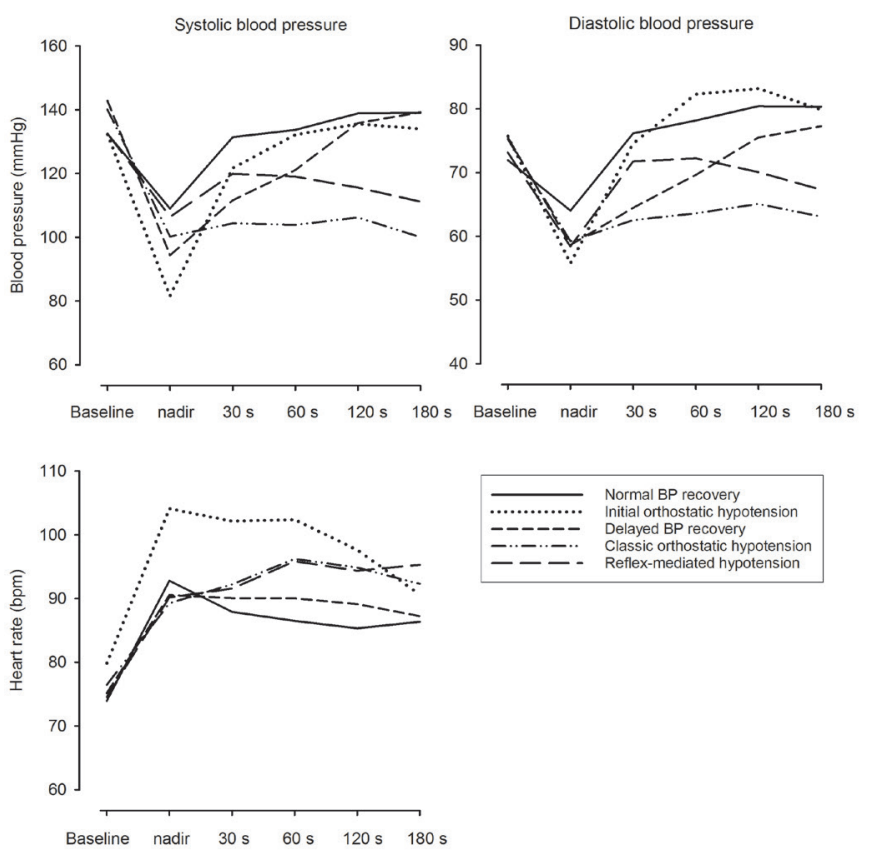

Figure 2 Haemodynamic profiles of the different orthostatic BP recovery patterns. Continuous non-invasive orthostatic BP measurement in 111 patients with (pre)syncope. Median BP and HR values are presented at baseline, nadir, 30, 60, 120 and 180s after standing up. Orthostatic BP recovery patterns are classified as normal BP recovery $(n=63)$, initial orthostatic hypotension $(n=8)$, delayed BP recovery $(n=14)$, classic orthostatic hypotension $(n=21)$ and reflex-mediated hypotension $(n=5)$. fibrillation $(n=4)$. The management of patients with presyncope and syncope, respectively, consisted of admission (16\% vs 21\%), referral to an outpatient department (49\% vs $20 \%$ ), referral to the general practitioner $(11 \%$ vs $9 \%)$ or no specific management (24\% vs $50 \%)$.

\section{DISCUSSION}

In this study, we found that $43 \%$ of the patients with (pre) syncope had an abnormal orthostatic BP recovery pattern. Classic orthostatic hypotension (19\%) was most frequently detected, followed by delayed BP recovery (13\%), initial orthostatic hypotension (7\%) and reflex-mediated hypotension (4\%). The aim of this study was to describe the frequency of abnormal orthostatic $\mathrm{BP}$ recovery patterns that cannot be captured by oscillometric BP measurement.

Current research in the ED is directed towards risk stratification associated with syncope. ${ }^{89}$ Thereby, two important elements are considered: risk of death and life-threatening events and risk of recurrence and physical injury. ${ }^{1}$ With increasing age, syncope secondary to underlying cardiovascular disease becomes more common. ${ }^{10}$ Besides structural heart disease as a major risk factor for sudden cardiac death and overall mortality, classic orthostatic hypotension is associated with a twofold higher risk of death owing to the severity of comorbidities. ${ }^{1}$ Based on recent studies, similar risks are prevalent in subjects with delayed BP recovery. ${ }^{3}$ Furthermore, to prevent recurrences, correct identification of the cause of the (pre)syncopal episode and appropriate treatment and advices are important.

Classic orthostatic hypotension was the most prevalent abnormal orthostatic BP recovery pattern on continuous monitoring, with a three times higher prevalence than in the general population. ${ }^{11}$ In this study, only $61 \%$ of patients classified as having orthostatic hypotension by the attending physician had a positive continuous BP test for classic orthostatic hypotension, while $48 \%$ of patients with a positive test for classic orthostatic hypotension received a different working diagnosis from the attending physician. There are several factors that can explain this difference. Either the diagnosis was given without 
Table 2 Syncope classification by attending physician versus orthostatic BP recovery pattern

\begin{tabular}{|c|c|c|c|c|c|c|}
\hline $\begin{array}{l}\text { Diagnosis by attending } \\
\text { physician, } \mathrm{n}(\%)\end{array}$ & All patients $(n=111)$ & $\begin{array}{l}\text { Normal } \\
\text { BP recovery }(n=63)\end{array}$ & $\begin{array}{l}\text { Initial orthostatic } \\
\text { hypotension }(n=8)\end{array}$ & $\begin{array}{l}\text { Delayed } \\
\text { BP recovery }(n=14)\end{array}$ & $\begin{array}{l}\text { Classic orthostatic } \\
\text { hypotension }(n=21)\end{array}$ & $\begin{array}{l}\text { Reflex-mediated } \\
\text { hypotension }(n=5)\end{array}$ \\
\hline Vasovagal reflex syncope & $45(41)$ & $31(69)$ & $1(2)$ & $3(6)$ & $7(16)$ & $3(7)$ \\
\hline Situational reflex syncope & $9(8)$ & $5(56)$ & $1(11)$ & $2(22)$ & $1(11)$ & - \\
\hline Orthostatic hypotension & $18(16)$ & $4(22)$ & - & $1(6)$ & $11(61)$ & $2(11)$ \\
\hline Cardiac syncope & $10(9)$ & $6(60)$ & $2(20)$ & $2(20)$ & - & - \\
\hline Unexplained syncope & $29(26)$ & $17(59)$ & $4(14)$ & $6(21)$ & $2(6)$ & - \\
\hline
\end{tabular}

Classification of the cause of syncope diagnosed by the attending physician. The attending physician performed his usual syncope evaluation in the ED. Conclusive information about the orthostatic BP recovery patterns was not available at the time of diagnosis.

performing orthostatic BP measurement or measurement result was not taken into account or the test was not performed correctly or it was inaccurate. The prevalence of classic orthostatic hypotension as the cause of syncope in the literature lies between $4 \%$ and $24 \%$ and probably depends on whether the test has been performed (accurately) and the age of the population. ${ }^{12}$ Correctly classifying classic orthostatic hypotension is important because of the short-term risk of recurrences and falling and long-term association with cardiovascular morbidity and mortality. ${ }^{31013}$ Classic orthostatic hypotension can be caused by primary or secondary autonomic failure, it can be drug induced (eg, vasodilators, antidepressants) or caused by volume depletion. ${ }^{1}$ Treatment and advice are dependent on the underlying cause. ${ }^{13}$

It is only recently that delayed BP recovery has been recognised as a cause of unexplained falls and (pre)syncope and has been associated with similar short-term and long-term risks as classic orthostatic hypotension. ${ }^{3}{ }^{10}$ In the present syncope guidelines by the European Society of Cardiology and by ACC/ AHA/HRS, delayed BP recovery, as a variant of orthostatic hypotension, is not mentioned. ${ }^{14}$ In our population, $43 \%$ of the patients with delayed BP recovery were classified as unexplained syncope. Compared with those with classic orthostatic hypotension, patients with delayed BP recovery were older, had higher prevalence of hypertension and had a higher admission rate. Delayed BP recovery can be seen as a physical sign of subclinical impaired physiology. ${ }^{3}$ Therefore, we suggest that (older) patients presenting to the ED with a history of orthostatic (pre) syncope shortly after standing up, but with a negative oscillometric orthostatic BP measurement, be referred to a syncope unit to detect the underlying cause. ${ }^{15}$

Initial orthostatic hypotension was present in eight $(7 \%)$ patients in our study. This is a common (3\%-10\%) but unrecognised cause of syncope usually seen in younger patients. ${ }^{16}$ The clinical diagnosis is based on a typical history of (pre)syncope directly on standing and no classic orthostatic hypotension during oscillometric orthostatic BP measurement. ${ }^{17}$ The diagnosis becomes $100 \%$ certain with detection of initial orthostatic hypotension during continuous non-invasive BP measurement and recognisable symptoms. Recognition by the physician is important because management is simple and effective, that is, get up slowly and clench the buttocks (counterpressure manoeuvres). ${ }^{16}{ }^{17}$ When these manoeuvres abort recognisable symptoms in the patient, the diagnosis is confirmed. ${ }^{17}$

\section{LIMITATIONS}

The number of patients within the different orthostatic BP recovery patterns is relatively small. Nonetheless, this study was observational and primarily intended to determine the frequency of several forms of orthostatic (pre)syncope. Furthermore, continuous BP measurements were only performed during workdays. Nevertheless, the classification of syncope by the attending physician (table 2) was similar to other studies performed in the ED. ${ }^{1}$ Although we found that many patients with (pre)syncope had indeed an abnormal BP pattern, further assumptions about potential misdiagnosis by the attending physician could not be made, because this was not part of the study protocol. Moreover, we did not observe orthostatic BP measurements by the attending physicians and can therefore not state whether this was performed correctly or whether orthostatic testing was absent. Currently, we are investigating whether continuous orthostatic BP measurement in the ED is of added value in addition to extensive history taking.

\section{CONCLUSION}

Continuous non-invasive BP measurement can potentially identify more specific and concerning causes of (pre)syncope than separate oscillometric measurements. Correct classification is important because of the short-term and long-term clinical implications.

Contributors VKvW, DTH, WN, JCTM and MPMH conceived the study and designed the study protocol. JCTM acquired the waiver from the Institutional Review Board. VKvW and DTH performed the measurements and VKvW, DH, MPMH and AMvR performed the analysis. ROBG critically revised the manuscript. All authors contributed to the final draft of the manuscript.

Competing interests None declared.

Ethics approval Medische Ethische Toetsingscommissie Groningen.

Provenance and peer review Not commissioned; externally peer reviewed.

Open Access This is an Open Access article distributed in accordance with the Creative Commons Attribution Non Commercial (CC BY-NC 4.0) license, which permits others to distribute, remix, adapt, build upon this work non-commercially, and license their derivative works on different terms, provided the original work is properly cited and the use is non-commercial. See: http://creativecommons.org/ licenses/by-nc/4.0/

(C) Article author(s) (or their employer(s) unless otherwise stated in the text of the article) 2018. All rights reserved. No commercial use is permitted unless otherwise expressly granted.

\section{REFERENCES}

1 Moya A, Sutton R, Ammirati F, et al. Guidelines for the diagnosis and management of syncope (version 2009). Eur Heart J 2009;30:2631-71.

2 van Wijnen VK, Finucane C, Harms MPM, et al. Noninvasive beat-to-beat finger arterial pressure monitoring during orthostasis: a comprehensive review of normal and abnormal responses at different ages. J Intern Med 2017;282:468-83.

3 Lagro J, Schoon Y, Heerts I, et al. Impaired systolic blood pressure recovery directly after standing predicts mortality in older falls clinic patients. J Gerontol A Biol Sci Med Sci 2014;69:471-8

4 Nowak RM, Sen A, Garcia AJ, et al. Noninvasive continuous or intermittent blood pressure and heart rate patient monitoring in the ED. Am J Emerg Med 2011;29:782-9.

5 Peeters SY, Hoek AE, Mollink SM, et al. Syncope: risk stratification and clinical decision making. Emerg Med Pract 2014;16:1-22. quiz 22-3.

6 Grossman SA, Babineau M, Burke L, et al. Do outcomes of near syncope parallel syncope? Am J Emerg Med 2012;30:2064-5. author reply 2064. 
7 Romero-Ortuno R, O'Connell MD, Finucane C, et al. Insights into the clinical management of the syndrome of supine hypertension-orthostatic hypotension (SHOH): the Irish Longitudinal Study on Ageing (TILDA). BMC Geriatr 2013;13:73.

8 Costantino G, Sun BC, Barbic F, et al. Syncope clinical management in the emergency department: a consensus from the first international workshop on syncope risk stratification in the emergency department. Eur Heart J 2016;37:1493-8

9 Toarta C, Mukarram M, Arcot K, et al. Syncope Prognosis Based on Emergency Department Diagnosis: A Prospective Cohort Study. Acad Emerg Med 2017 doi: 10.1111/acem. 13346 (Epub ahead of print: 14 Nov 2017).

10 Finucane C, O'Connell MD, Donoghue 0 , et al. Impaired orthostatic blood pressure recovery is associated with unexplained and injurious falls. J Am Geriatr Soc 2017:65:474-82.

11 Finucane C, O'Connell MD, Fan CW, et al. Age-related normative changes in phasic orthostatic blood pressure in a large population study: findings from The Irish Longitudinal Study on Ageing (TILDA). Circulation 2014;130:1780-9.
12 Sarasin FP, Louis-Simonet M, Carballo D, et al. Prevalence of orthostatic hypotension among patients presenting with syncope in the ED. Am J Emerg Med 2002;20:497-501.

13 Ricci F, De Caterina R, Fedorowski A, et al. Orthostatic hypotension: epidemiology, prognosis, and treatment. J Am Coll Cardiol 2015;66:848-60.

14 Shen WK, Sheldon RS, Benditt DG, et al. 2017 ACC/AHA/HRS guideline for the evaluation and management of patients with syncope: a report of the American College of Cardiology/American Heart Association Task Force on Clinical Practice Guidelines and the Heart Rhythm Society. Heart Rhythm 2017; 14:e155-217.

15 Kenny RA, Brignole M, Dan GA, et al. Syncope unit: rationale and requirement - the European Heart Rhythm Association position statement endorsed by the Heart Rhythm Society. Europace 2015;17:1325-40.

16 Wieling W, Krediet CT, van Dijk N, et al. Initial orthostatic hypotension: review of a forgotten condition. Clin Sci 2007:112:157-65.

17 van Wijnen VK, Harms MP, Go-Schön IK, et al. Initial orthostatic hypotension in teenagers and young adults. Clin Auton Res 2016;26:441-9. 